\title{
CAN THE SOLAR SYSTEM BE QUANTIZED?
}

\author{
J. M. BARNOTHY
}

833 Lincoln Street, Evanston, Ill., U.S.A.

\begin{abstract}
It is suggested that the general form of the constant of quantization, $K$ in Schrödinger's equation, is not $h / 2 \pi$, but $K=2 s \alpha^{-k}$, with $s$ being the spin of the orbiting object, $\alpha$ the fine structure constant $(1 / 137.0361)$, and $k$ a small positive integer, or zero. For atoms $k=0$; for planets and satellites $k=2,3$ or 4 ; for the solar system as a whole, revolving around the center of the Galaxy, $k=6$. The probability that 16 objects of the solar system would follow this quantum rule by chance alone is 1 in $10^{16}$, suggestive that quantum mechanics, as we know it today, can be seen as a special case of a more general quantum mechanics of the future; it also supports the view expressed by Dirac, that $h$ is probably not a fundamental constant.

Section 1 contains the basic idea which induced me to undertake an investigation of a relationship between rotational and orbital angular momenta of planets; Sections 2-7 contain the experimental data, the application of the new quantum rule and the statistical evaluation whether the relationship proposed in Section 1, could have occurred by chance alone. The results obtained in Sections 2-7 are noteworthy in themselves, independently whether the basic idea is accepted or not.
\end{abstract}

February 1973 is the quinquecentennial anniversary of the birth of Copernicus, who recognized that the planets revolve around the Sun, and in doing so revolutionized the premises of all subsequent astronomy and cosmology. The year 1973 also marks the 60 th anniversary of Bohr's discovery of his atomic model: electrons revolve on Keplerian orbits around a nucleus, like planets around the Sun. Is there a further physical law common to both?

\section{A New Quantum Rule}

Let us ask the question what would have happened to quantum theory if the electron spin $\left(s_{e}\right)$ had been known in 1900 ? Let us take a ride in a time machine capable of rearranging the sequence of events.

We start with Goudsmith and Uhlenbeck's discovery of the electron spin. Millikan and Einstein show that in the photoelectric effect light is absorbed in quanta, each quantum carrying an energy $4 \pi s_{e} v$. At the next stop of our journey we find Planck saying that oscillators do not emit light continuously but in quanta, with energies as given by Einstein's equation. Obviously a light quantum sometimes behaves as a wave, and other times as a corpuscle.

Then the Broglie recognizes that since nature seems to have a preference for symmetries, if light behaves in a dualistic manner, then particles should also behave at times undulatorily; whereby, in analogy with light quanta, particle waves should also exist with wavelength equal to the ratio of $4 \pi$ times the spin of the particle to its momentum. Next Bohr explains the discrete set of Balmer lines, by postulating that electrons revolving around the nucleus do not emit radiation as long as they move on orbits, along which the de Broglie waves are standing waves. This means, of course, that the 
orbital angular momentum of the electron must always be an integer multiple of the electron spin. Unfortunately Heisenberg shows that the simultaneous observation of conjugate quantities always involves an error equal to the electron spin, hence it is impossible to observe Bohr's orbits. Schrödinger saves the situation by setting up a partial differential equation, leading for negative energies to a discrete set of Eigenvalues. He achieves this by substituting in the Hamiltonian a new variable $S=K \ln \psi$. To remain in agreement with observations, he sets the numerical value of $K$ equal to twice the spin of the electron.

We have now covered the events leading up to Schrödinger without the need to mention $h$, that is, Planck's constant. This curious circumstance raised in the ' $40 \mathrm{~s}$ the heretical thought (Barnothy 1946) in me that $h$ is probably not a fundamental constant of nature, but a constant, the value of which changes in accordance with the spin of the orbiting object. I found that the general formula for the constant $K$ in Schrödinger's equation would then be:

$$
K=2 s_{x} \alpha^{-k},
$$

where $s_{x}$ is the spin of the oscillating or revolving body, $k$ is a small positive integer or zero. To remain in agreement with observations, the constant $\alpha$ has to be set equal to the fine structure constant, $\frac{1}{137}$.

In the special case of the atom, $k=0$; and since $s_{x}=s_{e}, K=h / 2 \pi$ as assumed by Schrödinger. But in the case of the Earth $k=3$, and $s_{x}$ is the spin of the Earth, consequently 'Planck's constant' according to which the orbit of the Earth should be quantized is $1.7 \times 10^{45} \mathrm{cgs}$. Since the Earth is in its lowest quantum state, its de Broglie wavelength equals the circumference of the ecliptic. It is conceivable that when the Earth was formed, it occupied a higher quantum state, and by releasing energy in the form of gravitational waves it 'jumped' to its present 'ground state'. I do not wish as yet to speculate further on a possible gravitational wave spectrum emitted from newly formed planetary systems in the universe.

\section{Application to the Planetary System}

The formula to compute $n$, the quantum number of the planets, is:

$$
n=\frac{\text { orbital angular momentum }}{2 \text { rotational spin }} \alpha^{k}=(t / T)(R / r)^{2} \times 137.04^{-k}(2 \tau \cos \delta)^{-1},
$$

where $r$ and $t$ are radius and period of rotation (in tropical days) of the planet; $T$ and $R$ the orbital period and distance from the Sun, respectively; $\tau=I / M r^{2}$ is the moment of inertia factor; and $\delta$ the inclination of the equator to the orbital plane.

No actual reason can be given for the occurrence of the factor $\alpha$ in Equations (1) and (2). But we should remember that the fine structure constant appears unexplanably in many places in physics. Some theoretical physicists believe that the fine structure constant may have something to do with geometrical calculations. Such an approach 
was suggested by Barnothy (1947) and recently by Wyler (1969). Barnothy's approach to the problem is based on an application of Friedmann's field equations to nuclear forces, leading to a geometrical structure of elementary particles, which in turn permits the exact calculation of dimensionless numbers such as $\alpha$, gyromagnetic ratios, and the mass ratio of proton and electron. Wyler's approach is purely geometrical and compares the volume elements of a seven-dimensional group having five real and two imaginary timelike dimensions, with those of the subgroup of the five real dimensions.

Table I shows the quantum levels of the nine planets. Three different $k$ values were

TABLE I

Quantum levels of major planets

\begin{tabular}{|c|c|c|c|}
\hline & $k$ & $n$ & $\Delta[n]$ \\
\hline Mercury & 4 & 4.15 & +0.15 \\
\hline Venus ${ }^{a}$ & 4 & 0.69 & -0.31 \\
\hline Earth & 3 & 0.97 & -0.03 \\
\hline Mars & 3 & 3.83 & -0.17 \\
\hline Jupiter & 2 & 1.30 & -0.30 \\
\hline Saturn & 2 & 3.14 & +0.14 \\
\hline Uranus ${ }^{\mathbf{a}}$ & 3 & 0.99 & -0.01 \\
\hline Neptune & 3 & 0.29 & - \\
\hline Pluto & 4 & 0.91 & -0.09 \\
\hline
\end{tabular}

Retrograde rotation.

needed. Among the nine planets, only Neptune does not fit into the scheme. When two planets occupy the same $n$ and $k$ quantum numbers - e.g. Pluto and Venus, and again Earth and Uranus - one of them happens to have retrograde rotation, suggestive that Pauli's exclusion principle might be applicable.

The probability to find among nine planets eight with integer $n$ values [1], [2], [3] and [4] by chance alone is 1 in $10^{8}$. (For the computations see Section 5.) Such a small probability suggests that Equation (1) may in fact be a generally valid formula to compute the factor of quantization $(K)$ in Schrödinger's equation.

This in turn leads to four further inferences:

(1) It is possible to quantize macroscopic rotators.

(2) Planck's constant is not a fundamental physical constant.

(3) The uncertainty relation depends on what kind of signal carriers we use. Photons give very blurred pictures of Bohr's orbits, but very sharp pictures of the orbits of the planets. Gravitational waves, on the other hand, would give very hazy pictures of the planetary system, and what one could say would merely be that the probability to find the Earth somewhere around the Sun is proportional to $\psi^{2}$. This means that model-like descriptions should not be dismissed as worthless: they are complementary to statistical mathematical descriptions. Nature appears dualistic even in its recognition patterns (Barnothy, 1947). 
(4) Should the above considerations be correct, their effect on present quantum mechanics would be similar to Einstein's special relativity on Newtonian mechanics, current quantum mechanics being a special case of a more general quantum mechanics of the future.

\section{Observational Data}

The orbital parameters of the planets and satellites are rather precisely known, while radii, periods of rotation, and moment of inertia factors $(\tau)$ are occasionally uncertain. For the latter the best values as reported in Dollfus (1970), Surfaces and Interiors of Planets and Satellites, were used. In the following I refer to this book as SIPS, indicating authors and page number. The used values are listed in Table III.

According to Plagemeann's (1965) thermal calculations (see also Majeva, 1969) the interior of Mercury was never molten, hence a coreless model, with uniform distribution of metallic iron must be preferred (Levin in SIPS, p. 481), with a moment of inertia factor $\tau=0.388$, at a radius of $r=2432 \pm 7 \mathrm{~km}$ (Dollfus in SIPS, p. 136). The sidereal period of rotation of Mercury is 58.65 days (Dyce in SIPS, p. 160), $\frac{2}{3}$ times its orbital period. One solar day on Mercury (which we shall call the tropical period of rotation of the planet or satellite) is $t=\left(t_{s}^{-1}-T^{-1}\right)^{-1}=176.01$ days.

The three-zone model of Venus, consisting of a rock crust, a metallized silicon layer and a metallic iron core is probably correct (Levin in SIPS, p. 493 and 495). We adopt $\tau=0.341$ and $r=6052 \pm 6 \mathrm{~km}$ (Dollfus in SIPS, p. 136). The recent radar observations of Shapiro (1967) give a sidereal period of rotation in astounding agreement with the Earth-Venus resonance value of $\mathbf{2 4 3 . 1 6}$ days. Considering the retrograde rotation of Venus, this corresponds to a tropical period of rotation of $t=\left(t_{s}^{-1}+T^{-1}\right)^{-1}=116.78$ days.

The moment of inertia of the Earth is well established from mass distribution models and from its hydrostatic flattening, yielding $\tau=0.3309$.

Several models have been developed for Mars, but each of them was fitted to the observed flattening, hence they cannot yield additional information regarding the moment of inertia factor of this planet. The observations of Phobos and Deimos yield $\tau=0.375$ (Levin in SIPS, p. 487).

No reliable models are available for Jupiter, Saturn, Uranus and Neptune. I have computed their moment of inertia factor $(\tau)$ as for Mars from the observed hydrostatic flattening $(f)$ and the mass $(M)$ of the planet, using the equation:

$$
\tau=\frac{2}{3}\left[1-\frac{2}{5}(5 m / 2 f-1)^{1 / 2}\right],
$$

where $m=4 \pi^{2} r^{3} / G t_{s}{ }^{2} M$ is the ratio of centrifugal to gravitational accelerations on the equator of the planet. Such a computation of the moment of inertia factor around the polar axis is based on the assumption that the surface of the planet is an equipotential surface. Table II lists the used equatorial and polar radii, the $f$ and $M$ and the resulting $\tau$ values.

The flattening of Uranus computed from the optically observed equatorial and polar diameters leads to an impossibly small $\tau$ value. Cook (1972) believes that a 
flattening between $f=4.5 \times 10^{-2}$ and $5.5 \times 10^{-2}$ would be reasonable. Kovalevsky (in SIPS, p.32) suggests a flattening of $\frac{1}{18}$. I have accepted this latter value in my computations.

The difference between the equatorial and the polar radii of Neptune is not known. The coefficient of the second harmonic in the gravitational potential of Neptune has been derived from the motion of the satellite Triton, with the result $J_{2}=5 \times 10^{-3}$ (Kovalevsky in SIPS, p. 39). If the surface of the planet is assumed to be equipotential,

TABLE II

Moment of inertia factor

\begin{tabular}{llllll}
\hline & $\begin{array}{l}r_{e} \\
(\mathrm{~km})\end{array}$ & $\begin{array}{l}r_{p} \\
(\mathrm{~km})\end{array}$ & $f$ & $\begin{array}{l}M \\
\left(10^{24} \mathrm{~g}\right)\end{array}$ & \\
\hline Jupiter & $70850^{\mathrm{a}}$ & $66550^{\mathrm{a}}$ & 0.0607 & $1900^{\mathrm{b}}$ & 0.233 \\
Saturn & $60000^{\mathrm{a}}$ & $53450^{\mathrm{a}}$ & 0.1092 & $569.3^{\mathrm{b}}$ & 0.213 \\
Uranus & $25400^{\mathrm{a}}$ & $24700^{\mathrm{a}}$ & 0.0555 & $87.7^{\mathrm{c}}$ & 0.263 \\
Neptune & $25225^{\mathrm{d}}$ & & $0.0217^{\mathrm{b}}$ & $102.9^{\mathrm{e}}$ & 0.264 \\
\hline
\end{tabular}

a Dollfus in SIPS, p. 136.

b Kovalevsky in SIPS, pp. 31, 39.

c Klepcynski et al., 1970.

d Kovalevsky and Link, 1969.

- Seidelmann et al., 1969.

the polar flattening is given, to first order, by the formula $f=\frac{3}{2} J_{2}+\frac{1}{2} m$, whence the flattening is $2.17 \times 10^{-2}$.

Among all the planets Pluto has the most eccentric orbit, which moreover intercepts the orbit of Neptune. This is one of the reasons to regard Pluto as an escaped satellite. Its size and density, however, place it in the class of terrestrial planets. Recently all available transit circle observations of Neptune, from its discovery in 1846 to the present, have been reexamined by Seidelmann et al. (1971). Pluto's mass was found to be $(0.107 \pm 0.001) M_{\oplus}$ very close to that of Mars. We adopt, therefore, for the moment of inertia factor of Pluto the same value $\tau=0.375$ as for Mars, and take its radius to $3200 \mathrm{~km}$, the value used by Seidelmann et al.

We may assume that the very small asteroids behave as rigid bodies, whence $\tau$ reaches for spherically shaped objects its maximal value of $\frac{2}{5}=0.40$. Only two asteroids, Ceres and Vesta, could be included in Table III. The period of rotation of Pallas and the diameter of Juno and Eros are too uncertain; for the others only orbital data are known.

The moment of inertia factor of the Moon was very exactly determined to $0.3906 \pm$ \pm 0.0003 from the orbits of the many artificial satellites which during the last years orbited the Moon (Tolson et al., 1967). The same $\tau$ value was adopted for all the other satellites.

The resulting quantum numbers $k$ and $n$ of 9 planets, 2 asteroids and 8 satellites are listed in the last columns of Table III. 
TABLE III

Quantum levels of major and minor planets and satellites

\begin{tabular}{|c|c|c|c|c|c|c|c|c|c|}
\hline & $\begin{array}{l}t \\
\text { (days) }\end{array}$ & $\begin{array}{l}T \\
\text { (days) }\end{array}$ & $\begin{array}{l}r_{e} \\
(\mathrm{~km})\end{array}$ & $\begin{array}{l}R \\
\left(10^{8} \mathrm{~km}\right)\end{array}$ & $\tau$ & $\begin{array}{l}\delta \\
\text { (degree) }\end{array}$ & $k$ & $n$ & $\Delta[n]$ \\
\hline Mercury & 176.01 & 87.96 & $2432 \pm 7$ & 0.579 & 0.388 & 0 & 4 & 4.15 & +0.15 \\
\hline Venus $^{\mathrm{a}}$ & 116.78 & 224.69 & $6052 \pm 6$ & 1.082 & 0.341 & 1.2 & 4 & 0.69 & -0.31 \\
\hline Earth & 1 & 365.24 & $6370 \pm$ & 1.496 & 0.3309 & 23.45 & 3 & 0.97 & -0.03 \\
\hline Mars & 1.027 & 686.95 & $3402 \pm 8$ & 2.279 & 0.375 & 24.86 & 3 & 3.83 & -0.17 \\
\hline Jupiter & 0.4097 & 4332 & $70850 \pm 100$ & 7.782 & 0.233 & 3.083 & 2 & 1.30 & +0.30 \\
\hline Saturn & 0.4264 & 10796 & $60000 \pm 240$ & 14.30 & 0.213 & 26.81 & 2 & 3.14 & +0.14 \\
\hline Uranus $^{\mathrm{a}}$ & 0.450 & 30662 & $25402 \pm 280$ & 28.68 & 0.263 & 98 & 3 & 0.99 & -0.01 \\
\hline Neptune & 0.658 & 59863 & $25225 \pm 450$ & 44.81 & 0.264 & 27 & 3 & 0.29 & - \\
\hline Pluto & 6.375 & 90324 & $3200 ?$ & 58.93 & 0.375 & $?$ & 4 & 0.91 & -0.09 \\
\hline Ceres & 0.378 & 1682 & $385 \pm 20$ & 4.141 & 0.40 & $?$ & 4 & 0.92 & -0.08 \\
\hline Vesta & 0.223 & 1326 & $210 \pm 17$ & 3.532 & 0.40 & $?$ & 4 & 1.69 & -0.31 \\
\hline E. Moon & 29.53 & 365 & $1739 \pm 5$ & 1.496 & 0.3906 & $\sim 0$ & 4 & 2.17 & +0.17 \\
\hline J. Io & 1.77 & 4332 & $1750 \pm 75$ & 7.782 & 0.39 & 3 & 4 & 0.29 & - \\
\hline Europe & 3.55 & 4332 & $1550 \pm 75$ & 7.782 & 0.39 & 3 & 4 & 0.75 & -0.25 \\
\hline Ganymede & 7.14 & 4332 & $2775 \pm 65$ & 7.782 & 0.39 & 3 & 4 & 0.47 & - \\
\hline Callisto & 16.75 & 4332 & $2500 \pm 75$ & 7.782 & 0.39 & 3 & 4 & 1.36 & +0.36 \\
\hline S. Titan & 15.96 & 10796 & $2425 \pm 150$ & 14.30 & 0.39 & 27 & 4 & 2.10 & +0.10 \\
\hline Iapetus & 53.15 & 10796 & $575 \pm 50$ & 14.30 & 0.39 & 27 & 5 & 0.92 & -0.08 \\
\hline N. Triton ${ }^{b}$ & 5.88 & 59863 & $1885 \pm 750$ & 44.81 & 0.39 & $\sim 0$ & 4 & 2.03 & +0.03 \\
\hline
\end{tabular}

a Retrograde rotation.

b Retrograde orbit.

\section{Specific Questions}

One way to quantize the satellites would be to take their respective planets as central body. As far as we know, all satellites always turn the same side toward their mother planet, hence, their period of rotation is equal to their orbital period and Equation (2) is simplified to:

$$
n=(R / r)^{2} \alpha^{k}(2 \tau)^{-1} .
$$

However, the results one obtains from Equation (4) is a completely random dispersion of $n$ values, scattered between 2 and 50, indicating that satellites cannot be quantized in such a manner.

It seems to me that the explanation of this behavior is that the spin of the orbiting object has a physical meaning for quantization purposes only as related to the radius vector of its orbit. Satellites, which always turn the same side toward their planet, do not rotate relative to their radius vector, whence their spin is zero in the coordinate system of their orbit around the planet!

Satellites, however, rotate relative to the radius vector of their orbit around the Sun, and can be quantized in the same manner as planets, orbiting around the Sun, as this is illustrated in Table III. 
Artificial satellites (Apollo, Mariner, etc.) turn always the same side toward the Moon, or the planet they orbit, thus their spin is zero relative to the radius vector of their orbit. Similarly as celestial satellites, they cannot be quantized when orbiting a planet. During their passage from the Earth to their target, artificial satellites are stabilized relative to the direction of the Sun. Consequently, they cannot be quantized as being in orbit around the Sun either.

I could only include the Moon, four satellites of Jupiter and two satellites of Saturn in Table III, because the diameters of the other larger satellites are not yet known with sufficient accuracy. Although the diameter of Triton is uncertain to $\pm 40 \%$, nevertheless I included it because it is the second largest satellite, and because it has a retrograde orbit relative to its mother planet. There are three satellites in the $n=2, k=4$ quantum state, and only one has retrograde rotation; two satellites are in the $n=1, k=4$ quantum state, neither has retrograde rotation. This does not seem to support the applicability of Pauli's exclusion principle.

The circumstance that the rotational spin of the orbiting objects has to be computed relative to the radius vector of the orbit requires use of the tropical period of rotation, instead of the sidereal period of rotation. This difference becomes significant only for the planets Mercury and Venus, where the tropical periods of rotation are 176.01 and 116.78 days, instead of 58.65 and 243.16 sidereal days, respectively.

In 1946 when the periods of rotation of Mercury and Venus were not yet known, it was generally assumed that the period of rotation of these two innermost planets were the same as those of the two other terrestrial planets Earth and Mars that is, one day. Nevertheless, the quantum number $n$ which I computed at that time for Mercury and Venus was the same (Barnothy, 1946), as found now, that is 4 and 1, respectively. The quantum number $k$ was 3 instead of 4 .

This suggests that all four terrestrial planets were formed with the same $k=3$ value, Venus and Earth being then in the same quantum state $n=1$ and $k=3$, and Pauli's exclusion principle required that one member of the pair should have retrograde rotation. This was the reason why I concluded 30 years ago that Venus must have retrograde rotation, a prediction born out by later observations.

In the course of time the direction of rotation has not changed, but Mercury and Venus have increased their quantum number $k$ from 3 to 4 by lengthening their sidereal period of rotation by factors 56 and 244, respectively, changing thereby the original ratio of rotational to orbital angular momenta by a factor of about 137, without thereby changing their quantum level $n$. Mercury and Venus are much closer to the Sun than the Earth, and the solar tides are therefore much more powerful. It is generally assumed that tidal friction was the reason which increased the periods of rotation of the two innermost planets to their present rates. The astonishing agreement of the $n$ values, whether computed with one day, or with the present tropical periods of rotation, supports the assumption that at the start the period of rotation was the same for all four terrestrial planets.

The large deviation of the $n$ value of Venus from an integer is rather surprising, because its radius and moment of inertia factor are well known. We may seek an 
explanation in the phenomenon that Venus turns the same point of its surface toward the Earth at each inferior conjunction, indicating the existence of an Earth lock. It seems, therefore, possible that during the course of the slowing down of the rotation of Venus, this process was halted through the Earth lock.

It is generally assumed that spiral galaxies are systems in gravitation equilibrium, their star systems revolving on Keplerian orbits around the galactic center. The total angular momentum of the solar system is $s=3.21 \times 10^{50} \mathrm{cgs}$, while its mass is $2 \times 10^{33} \mathrm{~g}$. The distance to the Galactic center is $R=9.4 \mathrm{kpc}$ (Van den Bergh, 1972), and its rotational velocity $v=250 \pm 20 \mathrm{~km} \mathrm{~s}^{-1}$ (de Vaucouleurs and Peters, 1968). The equation to quantize the solar system revolving around the center of the Galaxy is:

$$
n=\frac{v R M}{2 s} \alpha^{k}(\cos \delta)^{-1}
$$

where $\delta=63.6^{\circ}$ is the inclination of the orbital plane of the major planets to the galactic plane. With $k=6$, we obtain $n=0.76$. The deviation from the integer number [1] could be due to an error of $30 \%$ in the distance from the Galactic center, but could also arise from the presence of a heavy trans-Plutonian planet with retrograde revolution. From the irregularities observed in the orbit of Neptune, Brady (1972) concluded upon the existence of a trans-Plutonian planet of $300 M_{\oplus}$ mass, with its orbital plane $120^{\circ}$ inclined to the ecliptic.

\section{Probability that $\boldsymbol{n}$ is an Integer Number by Chance Alone}

Altogether there are 19 objects ( 9 planets, 8 satellites and 2 asteroids) for which the radius $r$, period of rotation $t$, and moment of inertia factor $\tau$ are known with an accuracy better than $10 \%$ (see Table III). With the exception of Neptune, and two of the inner satellites of Jupiter, all the other 16 objects have $n$ values which deviate less than +0.36 and -0.31 from the integer numbers $1,2,3$ or 4 . If we compute $n$ from Equation (2) without the factor $\alpha^{k}$, then $n$ is of the order of $10^{4}$ to $10^{9}$. Applying the factor $\alpha^{k}$ divides the total range of $n$ in subintervals, each containing the same range of $n$ values from 1 to 137. Let us assume that we accept an $n$ value as being an integer quantum number if its deviation from [1], [2], [3] or [4] is less than \pm 0.25 . Then, the probability that a random number falls within \pm 0.25 from [1], [2], [3] or [4] would be $\frac{2}{137}=1.5 \%$. One has, however, to take into account that whenever $n$ is found to be a large integer, say between 103 and 137, this number could be changed into an $n$ value between 0.75 and 1 , simply by changing $\alpha^{k}$ to $\alpha^{k+1}$. We have thus to divide the $16 n$ values into two groups: one where $n$ is less than 1 , and another where $n$ is greater than 1 , the groups being populated by 7 and 9 objects, respectively. In the first group the chance of a success in one trial is about $\frac{137}{7} \sim 20$ times higher than in the second group.

The probability to find 7 objects in the first group and 9 in the second follows a biniminal distribution:

$$
P=\frac{N !}{x !(N-x) !} p^{x}(1-p)^{N-x}
$$


where $N$ is the number of trials, $x$ the number of successes (integer), and $p$ the probability for one trial. Here $p$ is determined by the average deviation of $n$ from integer values. Because the average deviation from [1] in the first group is $-0.11, p=0.11$ and with $x=7$ and $N=19$ we have

$$
P_{1}=\frac{19 !}{7 ! 12 !} 0.11^{7} \times 0.89^{12}=2.4 \times 10^{-3} \text {. }
$$

In the second group we have an average positive deviation of +0.33 for [1], but \pm 0.15 for [2], [3], or [4]; thus $p=(0.33+6 \times 0.15) 137^{-1}=9.0 \times 10^{-3}$. With $x=9$ and $N=19$,

$$
P_{2}=\frac{19 !}{9 ! 10 !}\left(9.0 \times 10^{-3}\right)^{9}=3.6 \times 10^{-14} .
$$

The total probability is thus:

$$
P=P_{1} P_{2}=8.6 \times 10^{-17} \text {. }
$$

The chance of 1 in $10^{16}$ cannot be called anymore a chance, but a proof that the solar system, hence macroscopic objects, can be quantized. Unless, of course, we want to believe that our solar system was specially selected by the Divine Creator from $10^{16}$ similar planetary systems to satisfy this curious agreement. This would be, however, equivalent to return to the old, pre-Copernician geocentric cosmological philosophy.

\section{Objections}

The possibility to launch artificial satellites in whatever orbits, contradicts the hypothesis of quantization of macroscopic objects. This objection is answered in Section 4. Even for a satellite rotating around an axis perpendicular to its orbit, it would take millions of years before its energy loss through gravitational radiation would suffice to settle it in a stable quantized orbit.

The angular velocity of the Earth is slowing down at a rate of $(2.65 \pm 0.58) \times 10^{-10}$ $\mathrm{yr}^{-1}$ (Newton, 1972). During the past life time of the Earth, this would have amounted to a considerable change in the ratio of the Earth's spin to its orbital angular momentum, contradicting quantization. A decrease of $0.000136^{\prime \prime} \mathrm{yr}^{-1}$ from the present $23^{\circ} 45^{\prime}$ inclination of the rotational axis to the normal of the ecliptic, would compensate the effect of the slowing down of the rotation of the Earth on the quantum number $n$, as this can be seen from Equation (2). On account of the wobbling of the polar axis by $\pm 0.3^{\prime \prime}$, the detection of such a quantum mechanical 'readjustement' of the rotational axis would be difficult to establish even within a century, although the six stations of the international network for latitude determinations are capable to determine the momentary direction of the polar axis to an accuracy of 0.01 ". 


\section{Further Consequences}

A quantization of binary systems consisting of two neutron stars reinstates the possibility that pulsars could be binary systems, because there is no emission of gravitational radiation while in stable orbits. To date some 20 different pulsar models were proposed. Some predict that the radiation is emitted from the first few centimeters thick surface layers of a neutron star, while in others the emission occurs at a height of $10000 \mathrm{~km}$. This indicates that in spite of the great sophistication of some models, the question of what pulsars are, is not finally settled. A few years ago I have proposed at the 127th meeting of American Astron. Soc. that should one be able to disregard for one reason or another, the slowing down process through the emission of gravitational radiation, a binary system of two neutron stars could explain without further assumptions many peculiarities of pulsars (Barnothy, 1968).

Quantization of a binary pulsar raises the hope that the 'glitches' in the pulse frequency of the Crab and Vela pulsars may be explanable through quantum jumps from a higher to a lower quantum state.

If the planetary system can be indeed quantized, it would entail a re-evaluation of the uncertainty principle, and with it, of quantum mechanics, certainly in its Copenhagen interpretation, which renounces reality as a metaphysical unscrutable. True, if an observer would look on our planetary system using gravitational waves with wavelength equal to those the system would emit when the planets would change from one quantum state to another, he could describe the position of the planets and their motion merely with the help of $\psi$-functions, and may thus say, that the existence of the Earth, for instance, is only a statistical probability. But, if he would use light, and would have a sufficiently powerful telescope to see people walking on the streets, I seriously doubt whether he would still question the physical reality of the Earth.

The solar system seems thus to be a quantized system, which depending on the signal carrier we use, obeys or rejects the uncertainty principle. The main importance of what I have here shown and hopefully proven is: the repudiation of John von Neumann's theorem, namely that no parameter previously 'hidden' from quantum physics could later be discovered and permit a precise measurement that violates the uncertainty law - thereby forever exiling cause-and-effect from the scene of physics.

Tycho Brahe's and Copernicus' astronomy could develop into modern astrophysics only with the help of physics. It seems that astronomy is now in the position to repay this debt by helping to understand the meaning of the discarded concepts of causality and dualism, concepts, though used for a long time, but understood only vaguely by the physicists themselves.

\section{References}

Barnothy, J.: 1946, Nature, June 15 and August 31.

Barnothy, J.: 1947, Papers of Terrestrial Magnetism, Hung. Inst. for Meteor. Terres. Magn., No. 2.

Barnothy, J.: 1968, Astron. J. 73, 164.

Brady, J.: 1972, Publ. Astron. Soc. Pacific 84, 314.

Cook, A. H.: 1972, Observatory 92, 84.

de Vaucouleurs, G. and Peters, L.: 1968, Nature 220, 368. 
Dirac, P. A. M.: 1963, Sci. Am. 208, No. 5, 45.

Dollfus, A. (ed.): 1970, Surfaces and Interiors of Planets and Satellites, Academic Press, New York.

Klepcynski, W. J., Seidelmann, P. K., and Duncombe, R. L.: 1970, Astron. J. 75, 739.

Kovalevsky, J. and Link, F.: 1969, Astron. Astrophys. 2, 398.

Majeva, S. S. V.: 1969, Astrophys. Letters 7, 11.

Newton, R. R.: 1972, Astrophys. Space Sci. 16, 179.

Plagemann, S.: 1965, J. Geophys. Res. 70, 985.

Seidelmann, P. K., Duncombe, R. L., and Klepcynski, W. J. : 1969, Astron. J. 74, 776.

Seidelmann, P. K., Klepcynski, W. J., Duncombe, R. L., and Jackson, E. S. : 1971, Bull. Am. Astron. Soc. 3, 270 .

Shapiro, I. I.: 1967, Science 157, 806.

Tolson, H. and Gapcinsky, J. P.: 1967, in A. Dollfus (ed.), Moon and Planets, II, Academic Press, New York, p. 178.

van den Bergh, S.: 1972, Dearborn Obs. Colloquium.

Wyler, A.: 1969, C.R. Acad. Sci. Paris 269A, 743. 